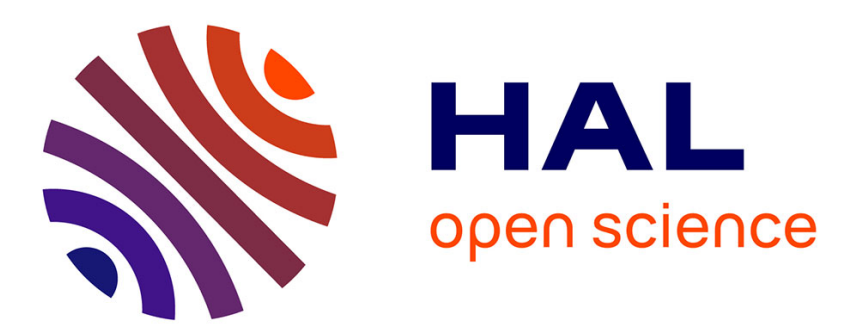

\title{
Controle de couches minces transparentes d'épaisseurs optiques quelconques application à des couches extrêmement minces
}

\author{
E. Pelletier, P. Giacomo
}

\section{- To cite this version:}

E. Pelletier, P. Giacomo. Controle de couches minces transparentes d'épaisseurs optiques quelconques application à des couches extrêmement minces. Revue de Physique Appliquée, 1967, 2 (1), pp.52-56. 10.1051/rphysap:019670020105200 . jpa-00242764

HAL Id: jpa-00242764

https://hal.science/jpa-00242764

Submitted on 1 Jan 1967

HAL is a multi-disciplinary open access archive for the deposit and dissemination of scientific research documents, whether they are published or not. The documents may come from teaching and research institutions in France or abroad, or from public or private research centers.
L'archive ouverte pluridisciplinaire $\mathbf{H A L}$, est destinée au dépôt et à la diffusion de documents scientifiques de niveau recherche, publiés ou non, émanant des établissements d'enseignement et de recherche français ou étrangers, des laboratoires publics ou privés. 


\title{
GONTROLE DE GOUGHES MINGES TRANSPARENTES D'ÉPAISSEURS OPTIQUES QUELGONQUES \\ APPLICATION A DES COUGHES EXTRÊMEMENT MINGES
}

\author{
Par E. PELletier, P. GIACOMO, \\ C.N.R.S., Laboratoire Aimé-Cotton, Bellevue, France. — Faculté des Sciences, Caen.
}

\begin{abstract}
Résumé. - On montre comment on peut utiliser le " maximètre " pour le contrôle pendant l'évaporation de couches non $\lambda / 4$ et en particulier de couches extrêmement minces. Deux méthodes sont proposées. La première, très simple et très générale, utilise un verre témoin; l'autre nécessite des calculs préalables difficiles mais permet le contrôle sur le verre à traiter lui-même. Ces méthodes permettent, avec une précision suffisante, le contrôle de couches d'épaisseur optique de l'ordre de $\lambda / 200$. Un exemple d'application est proposé : l'amélioration de planéité des lames d'interféromètre de Fabry-Perot.
\end{abstract}

Abstract. - It is shown how a "maximetre" may be used for monitoring thin films differing from $\lambda / 4$ in optical thickness, and specially extremely thin films. Two methods are proposed. The first one, very simple and general, uses a monitoring glass; the other one needs difficult preliminary calculations but allows monitoring on the sample itself. These methods enable films down to $\lambda / 200$ in thickness to be monitoring with considerable precision. An example application is proposed : improving the flatness of Fabry-Perot plates.

Introduction. - Les qualités de l'interféromètre de Fabry-Perot sont essentiellement limitées, dans l'état actuel, par les défauts de planéité des lames. On peut essayer d'améliorer cette planéité en déposant sur les lames une couche mince, transparente, d'épaisseur non uniforme. Le contrôle de l'épaisseur de cette couche pose un problème : la précision doit dépasser largement $\lambda / 100$. On sait que le « maximètre » [1] permet le contrôle précis de couches ou d'empilements de couches diélectriques d'épaisseur optique $\lambda / 4$. Nous nous sommes proposés d'utiliser sa précision pour le contrôle de couches d'épaisseurs quelconques et en particulier des couches d'épaisseurs très faibles, nécessaires dans ce cas. Nous verrons qu'il permet non seulement d'atteindre la précision nécessaire, mais encore d'effectuer le contrôle sur la lame elle-même.

\section{PREMIÈRE PARTIE CONTROLE DES EPAISSEURS}

I. Rappel du maximètre. - Le 《 maximètre » permet la localisation précise d'un maximum ou d'un minimum de transmission en fonction de la longueur d'onde.

Soit $y=f(\lambda)$ la variation du facteur de transmission ou de réflexion d'une lame transparente. Le maximètre permet d'observer une grandeur proportionnelle à la dérivée $\mathrm{d} y / \mathrm{d} \lambda$, et en particulier de repérer avec précision la valeur de $\lambda$ pour laquelle $\mathrm{d} y / \mathrm{d} \lambda$ s'annule. La lame dont on veut étudier la transmission est éclairée par une étroite bande spectrale de longueur d'onde $\lambda_{0}$. La longueur d'onde utilisée est modulée selon la loi :

$$
\lambda=\lambda_{0}+a \cos 2 \pi N t
$$

Le flux transmis est recueilli par une cellule photoélectrique dont le courant amplifié varie selon la loi :

$$
i=A y\left(\lambda_{0}+a \cos 2 \pi N t\right)
$$

Le coefficient $A$, fonction d'appareil, peut présenter lui-même une sélectivité en fonction de la longueur d'onde, due principalement à la source lumineuse et au récepteur photoélectrique employés.

L'amplitude de modulation étant petite, on peut développer suivant les puissances de $a$. On trouve une composante continue $A y\left(\lambda_{0}\right)$, puis une composante de fréquence $N$

$$
i_{1}=a[\mathrm{~d}(A y) / \mathrm{d} \lambda]_{\lambda_{0}} \cos 2 \pi N t
$$

d'amplitude relative :

$$
I_{1}=C a\left(\frac{1}{y} \frac{\mathrm{d} y}{\mathrm{~d} \lambda}+\frac{1}{A} \frac{\mathrm{d} A}{\mathrm{~d} \lambda}\right)
$$

$G$ est une constante qu'il est inutile d'expliciter ici. Lorsque $I_{1}$ s'annule, on a non pas $\mathrm{d} y / \mathrm{d} \lambda=0$ mais $\frac{1}{y} \frac{\mathrm{d} y}{\mathrm{~d} \lambda}=-\frac{1}{A} \frac{\mathrm{d} A}{\mathrm{~d} \lambda}$. Pour repérer avec précision les zéros de $\mathrm{d} y / \mathrm{d} \lambda$, il faut annuler $\mathrm{d} A / \mathrm{d} \lambda$ au voisinage de $\lambda_{0}$. On dispose sur un spectre intermédiaire du montage 
optique un « couteau » orientable, assurant une correction locale de $A(\lambda)$. Le réglage de l'orientation peut se faire en annulant le courant $i_{1}$ pour la longueur d'onde choisie en l'absence de l'objet à étudier.

$\mathrm{Si}$ on veut étudier la transmission d'un empilement quelconque de couches donné, la méthode de correction locale permet de déterminer par approximations successives les longueurs d'onde pour lesquelles $y(\lambda)$ est maximum (ou minimum). Le maximètre permet, plus facilement encore, de contrôler pendant l'évaporation des empilements de couches lorsque chacune d'elles a une épaisseur optique $p \lambda_{0} / 4$ ( $p$ entier). Il suffit de régler une fois pour toutes la correction locale. Nous allons montrer qu'il est également possible d'utiliser cet appareil pour la mesure de couches d'épaisseurs optiques quelconques.

II. Méthode de contrôle sur verre témoin. A) Principe. - On place côte à côte dans la cloche de l'évaporateur un verre témoin A qui servira pour le contrôle de l'évaporation et un verre B sur lequel on désire déposer une couche d'épaisseur optique ne. Un cache placé contre le verre $B$ permet de le masquer à volonté pendant une partie de l'évaporation.

On règle le maximètre sur une longueur d'onde $\lambda$ a priori quelconque; on règle ensuite le couteau pour annuler $\mathrm{d} A / \mathrm{d} \lambda$ pour cette longueur $\mathrm{d}$ 'onde; on évapore $q \lambda / 4$ sur le verre témoin A ( $q$ étant un nombre entier) en masquant soigneusement le verre $B$. On change ensuite de longueur d'onde de contrôle (en réglant à nouveau le couteau) et on poursuit l'évaporation jusqu'à $p \lambda^{\prime} / 4$ en évaporant simultanément sur le verre témoin et sur la lame à traiter. On aura ainsi déposé une épaisseur optique :

$$
n e=p\left(\lambda^{\prime} / 4\right)-q(\lambda / 4)
$$

sur la lame à traiter.

On dispose de quatre paramètres $\lambda, \lambda^{\prime}, p$ et $q$, ces deux derniers entiers. On peut donc non seulement donner à ne toute valeur imposée à l'avance, mais encore choisir trois des paramètres de telle façon que la sensibilité de la méthode soit maximale. Pour $\lambda$ et $\lambda^{\prime}$, on a intérêt à prendre des valeurs situées dans la zone de plus grande sensibilité du maximètre, cette zone est très large : elle n'est limitée que par le récepteur photoélectrique et la source. Pour $p$ et $q$, on sait que la sensibilité du maximètre augmente lorsque le nombre de couches $\lambda / 4$ déposées augmente. Mais, si $p$ ou $q$ deviennent très grands, les erreurs provenant de l'étalonnage en $\lambda$ deviennent prépondérantes. Il faut choisir un compromis. Avec l'appareil dont nous disposons, pour des couches optiques de l'ordre de $\lambda / 8$ à $\lambda / 200$, le meilleur compromis se situe vers $q=6$ à 8 .

Remarque : Ce calcul suppose implicitement que l'indice du corps considéré ne dépend pas de la longueur d'onde; si l'on connaît la dispersion, il est facile d'en tenir compte.
B) RÉglage du couteau. - Avant d'effectuer un dépôt sur le verre témoin, on règle le couteau de telle façon que $\mathrm{d} A / \mathrm{d} \lambda=0$ pour la première longueur d'onde. Il faut ensuite pouvoir annuler $\mathrm{d} A / \mathrm{d} \lambda$ pour la longueur d'onde $\lambda^{\prime}$. La variation de $A(\lambda)$ introduirait une erreur systématique inadmissible même si $\lambda$ et $\lambda^{\prime}$ ont des valeurs peu différentes. Le réglage pour la longueur d'onde $\lambda^{\prime}$ ne peut pas se faire à travers le verre témoin qui a déjà reçu une couche d'épaisseur $q \lambda / 4$. On a essayé de résoudre cette difficulté de deux façons.

1. On interpose sur le trajet lumineux un filtre de transmission $T(\lambda)=1 / A(\lambda)$. Un tel filtre serait extrêmement difficile à réaliser pour un grand domaine de longueur d'onde, mais pour un domaine restreint, le couteau permettant d'annuler les termes du premier ordre de la variation de la fonction d'appareil, il suffit que le filtre compense les termes du deuxième ordre. Étant donné la convexité de la fonction $A(\lambda)$, on peut dire a priori que le dépôt doit présenter un minimum de transmission pour une longueur d'onde voisine de $\lambda$; il peut être constitué par exemple d'une couche de haut indice d'épaisseur voisine de $k \lambda / 4 ; k$ étant un nombre impair à déterminer expérimentalement. On arrive ainsi à obtenir une compensation correcte dans un intervalle de $400 \AA$ autour de $\lambda=5000 \AA$. Cet intervalle est suffisant pour permettre de réaliser des couches d'épaisseurs quelconques sans avoir à régler le couteau lorsqu'on change de longueur d'onde. Malheureusement, par suite d'un vieillissement lent (vraisemblablement dû à l'état des miroirs placés à l'intérieur de la cloche de l'évaporateur), la compensation se détériore au cours du temps et il est nécessaire de refaire ce filtre périodiquement.

2. Il suffit en fait de pouvoir annuler la dérivée de la fonction d'appareil après chaque changement de longueur d'onde, en cours d'opération. On a disposé à cet effet, sur l'évaporateur, un système mécanique permettant de changer le verre témoin de position et de lui substituer un verre identique mais qui ne reçoit aucun dépôt. Le système est commandé de l'extérieur à travers un passage de type Wilson et le changement de verre témoin peut se faire dans le vide. Les verres étant disposés, pour raison d'uniformité, sur un support tournant pendant l'évaporation, cette dernière solution comporte quelques difficultés mécaniques; ces difficultés résolues, elle s'est avérée extrêmement pratique. On doit seulement vérifier avant les mesures que les verres ont bien une transmission identique : l'équilibrage du maximètre doit rester inchangé par permutation des deux verres.

C) RÉsultats. - A condition que la surface du verre témoin et celle de la lame à traiter soient de même nature (par exemple qu'elles aient été préalablement recouvertes d'une couche de même substance), cette méthode de contrôle donne de très bons résultats. 
Pour l'appliquer à la préparation d'empilements de couches de nature différente, il suffirait en principe de disposer d'un nombre de verres témoins supérieur d'une unité au nombre de substances différentes à déposer; le verre témoin supplémentaire est destiné au réglage du couteau. L'expérience montre alors que les épaisseurs déposées sur le verre à traiter et sur les verres témoins peuvent être très différentes. Cet effet est dû non seulement aux facteurs géométriques, mais surtout aux différences d'état de surface : on doit déposer sur chaque verre témoin une seule des substances à évaporer alors qu'on doit les alterner sur le verre à traiter; certains dépôts doivent donc, obligatoirement, être effectués sur des surfaces de natures différentes. Pour remédier à cet inconvénient, nous avons étudié la possibilité de contrôler le dépôt à travers la lame à traiter elle-même.

III. Méthode de contrôle à travers la lame à traiter. - Pour une lame recouverte d'un empilement de couches d'indices et d'épaisseurs donnés quelconques, le maximètre permet de déterminer la longueur d'onde pour laquelle l'empilement présente un extremum de pouvoir réflecteur : $\mathrm{d} R / \mathrm{d} \lambda=0$.

Réciproquement, on pourra contrôler l'épaisseur d'une couche que l'on désire ajouter sur un empilement existant si on connaît une longueur d'onde $\lambda^{\prime}$ pour laquelle l'empilement total, couche à rajouter comprise, présente un $\mathrm{d} R / \mathrm{d} \lambda=0$. Il suffira de régler le maximètre sur la longueur d'onde $\lambda^{\prime}$ correspondante, d'annuler $\mathrm{d} A / \mathrm{d} \lambda$ à l'aide $\mathrm{du}$ couteau et d'évaporer jusqu'à obtention $\mathrm{de} \mathrm{d} R / \mathrm{d} \lambda=0$.

Le principe est donc le même que celui utilisé pour le contrôle à travers le verre témoin, mais il nécessite un calcul préalable beaucoup plus difficile. En effet, lorsqu'on opère le contrôle sur une couche unique déposée sur verre témoin, on sait que les extremum de réflexion (ou de transmission) ont lieu pour les longueurs d'ondes pour lesquelles la couche a une épaisseur optique de $p \lambda / 4$. Lorsque l'empilement présente plusieurs couches d'épaisseurs variées, le calcul préalable des longueurs d'onde $\lambda, \lambda^{\prime}, \lambda^{\prime \prime}, \ldots$, pour lesquelles $R$ ou $T$ présentent des extremum, devient inabordable par les moyens usuels. Cette difficulté peut être résolue par l'emploi d'un simulateur [2].

Nous présenterons ici une application qui conduit à des calculs beaucoup plus simples : il s'agit de contrôler le dépôt de surépaisseurs sur un revêtement réflecteur constitué d'un nombre impair de couches, haut indice, bas indice alternées, chacune d'épaisseur optique $\lambda / 4$. Ce problème se présente lorsqu'on cherche à améliorer la planéité de lames d'interféromètre de Fabry-Perot.

Après avoir rapidement donné le principe de cette amélioration de planéité, nous exposerons les résultats obtenus quant au contrôle de surépaisseurs très faibles déposées sur le revêtement réflecteur.

\section{DEUXIEME PARTIE}

\section{APPLICATION A DES LAMES D'INTERFEROMETRE FABRY-PEROT}

Pour améliorer la planéité des lames, une solution séduisante consiste à boucher les trous de la lame par évaporation d'une quantité adéquate d'un matériau transparent [3]. Les deux lames de l'interféromètre sont repérées l'une par rapport à l'autre. On peut arbitrairement considérer que l'une des deux lames est parfaitement plane et déposer des couches correctrices uniquement sur l'autre.

I. Méthode d'amélioration des lames. - On établit une carte très précise des courbes de niveau de la lame à améliorer [4]; puis on place une des lames dans la cloche de l'évaporateur. On évapore alors selon un programme déterminé à partir de la carte obtenue. Supposons que l'on a relevé les courbes de niveau de $\lambda / n$ en $\lambda / n$ ( $n$ de l'ordre de 220 par exemple) et que

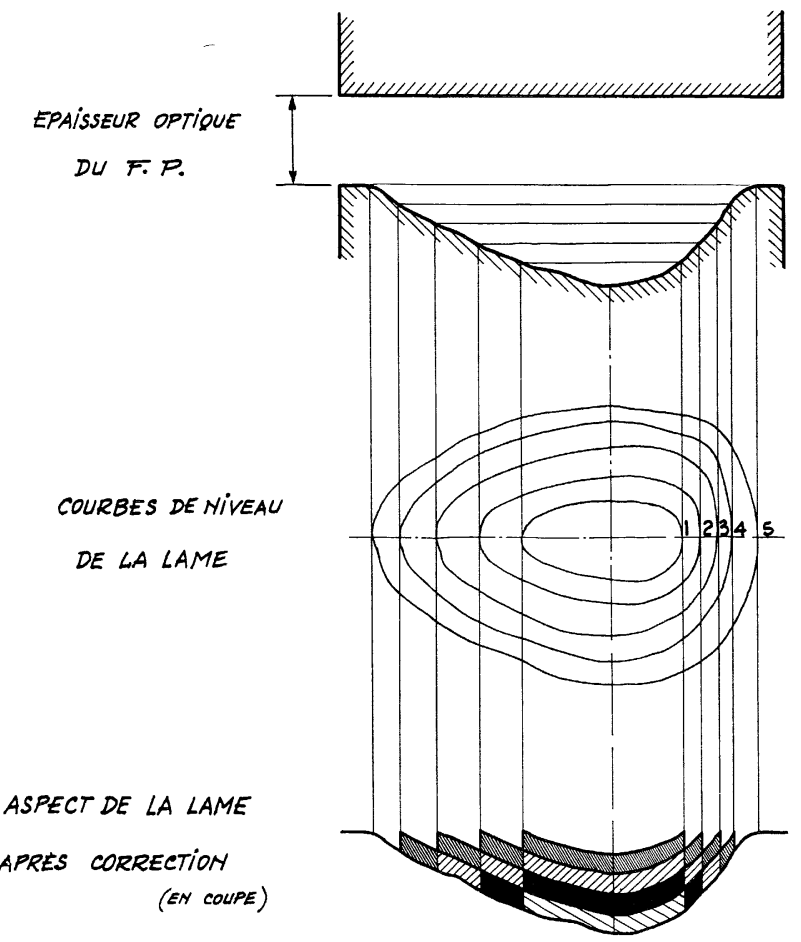

FIG. 1. - Principe de correction des lames.

le défaut est en forme de cuvette. Au départ, on place devant la lame un diaphragme dont l'ouverture reproduit la courbe de niveau 1 ( $f g .1$ ) et on commence l'évaporation. La substance évaporée se dépose seulement sur la partie de la lame découverte par le diaphragme. On évapore la quantité de matière nécessaire pour combler le fond de la cuvette. On enlève alors le diaphragme 1. C'est maintenant le diaphragme 2 qui limite la partie découverte de la lame, 
on dépose encore une surépaisseur et ainsi de suite. Finalement, la cuvette est comblée comme l'indique la figure 1. Les bords des couches successives seront marqués par des falaises de hauteur $\lambda / n$; elles seront adoucies par les effets de pénombre lors de l'évaporation.

La première idée qui vient à l'esprit est d'effectuer cette correction sur le support nu avec une substance de même indice. On ne dispose alors d'aucun procédé de contrôle satisfaisant pour le dépôt des couches correctrices : les contrôles sur la lame elle-même sont trop peu précis, et les contrôles sur verre témoin aléatoires. On peut, par contre, tirer profit du fait que, pour l'utilisation finale, les lames sont généralement recouvertes d'un revêtement réflecteur; dans la plupart des cas, on utilise un empilement de couches, haut indice, bas indice alternées, chacune d'épaisseur optique $\lambda / 4$.

Nous avons montré [5] que la correction sur la dernière couche du revêtement réflecteur présente plusieurs avantages.

1) Elle permet, au moins partiellement, de corriger des irrégularités éventuelles du revêtement réflecteur.

2) Les surépaisseurs à déposer sont très supérieures aux amplitudes des défauts à combler car les déphasages à la surface ont une influence prépondérante. Il est même nécessaire de déposer au préalable une couche uniforme, d'épaisseur optique $\lambda / 8$, pour que l'effet de la correction devienne appréciable.

3) En outre, il est plus commode d'ajouter des surépaisseurs au centre et les lames sont généralement convexes, il y a donc intérêt à pouvoir corriger une bosse en y ajoutant de la matière, ce qui est alors le cas.

Généralement les lames de Fabry-Perot ont une planéité optique de l'ordre de $\lambda / 40$ à $\lambda / 100 ;$ l'épaisseur optique totale des couches correctrices à déposer est de l'ordre de $\lambda / 20$ à $\lambda / 40$.

II. Contrôle des surépaisseurs. - Le problème que nous posons ici est de pouvoir, à travers la lame à traiter recouverte de son revêtement réflecteur, contrôler le dépôt d'une couche $\lambda / 8$ et d'un empilement d'une dizaine de couches, chacune ayant une épaisseur de $\lambda / 300$. Il s'agit donc simplement de mesurer ces surépaisseurs sur la dernière couche du revêtement.

Nous avons montré que pour pouvoir effectuer le contrôle il faut connaître la relation existant entre la surépaisseur optique $n \varepsilon$ de la dernière couche et la longueur d'onde $\lambda_{\mathrm{m}}$ pour laquelle $\mathrm{d} R / \mathrm{d} \lambda=0$. Soit $n \varepsilon=\varphi\left(\lambda_{\mathrm{m}}\right)$ cette relation. On peut la calculer numériquement en utilisant la méthode d'Abelès [6]. Ghaque couche est caractérisée par une matrice. Nous avons calculé, pour un empilement de sept couches (haut indice : sulfure de zinc, bas indice : cryolithe), toutes les couches ayant une épaisseur optique $\lambda_{0} / 4$, sauf la dernière qui a une épaisseur optique variable $n e=\left(\lambda_{0} / 4\right)+n \varepsilon$, le facteur de Fresnel $r$ pour l'amplitude en fonction de la longueur d'onde. On calcule ensuite le facteur de réflexion $R=r r^{*}$ pour l'intensité et l'on écrit la condition $\mathrm{d} R / \mathrm{d} \lambda=0$. On peut alors calculer numériquement $n \varepsilon=\varphi\left(\lambda_{\mathrm{m}}\right)$.

Ce calcul est beaucoup trop long pour être développé ici, on en trouvera un exposé plus détaillé dans une publication récente [7]. Nous donnerons simplement les résultats numériques obtenus. Sur la figure 2, on a porté en abscisses les valeurs de $\lambda_{\mathrm{m}} / \lambda_{0}$ et en ordonnées les valeurs calculées de $R$. Chaque courbe

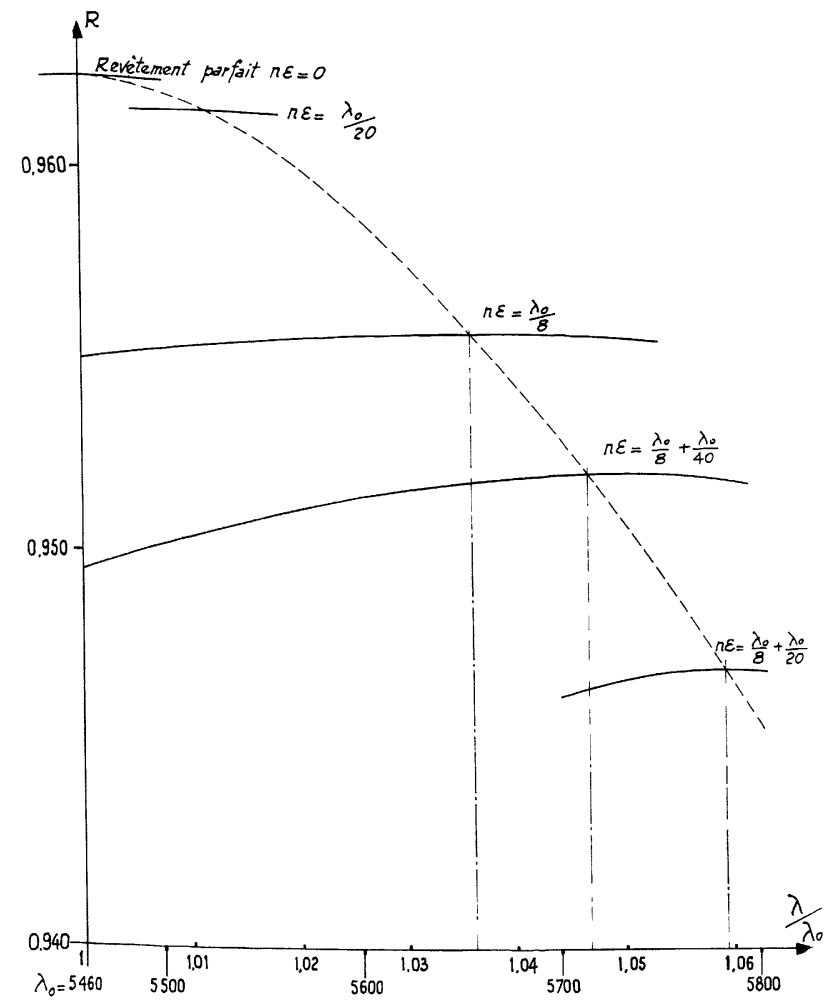

FIG. 2. - Pouvoir réflecteur en fonction de la longueur d'onde - Revêtement $7 \lambda_{0} / 4$ alterné ( $Z n S$ 'cryolithe) + surépaisseur optique $n \varepsilon(\mathrm{ZnS})$.

correspond à une surépaisseur optique de la dernière couche du revêtement qui est indiqué sur la figure. On obtient alors la forme de la fonction $\varphi: n \varepsilon=\varphi\left(\lambda_{\mathrm{m}}\right)$ (fig. 3).

On arrive aux conclusions suivantes :

L'empilement étant constitué de 7 couches $\lambda_{0} / 4$ avec $\lambda_{0}=5460 \AA$ par exemple :

- une surépaisseur $\lambda_{0} / 8$ déplace le maximum de $R$ de $197 \AA$;

- si on rajoute maintenant une couche $\lambda_{0} / 40$ le maximum se déplace de $58 \AA$.

Pour une surépaisseur $\lambda_{0} / 20$ on obtient un déplacement de $168 \AA$.

On a en moyenne un déplacement qui est de l'ordre de $12 \AA$ pour une surépaisseur $\lambda_{0} / 200$. Dans le montage actuel du maximètre existant au laboratoire, le monochromateur à réseau présente une dispersion largement 


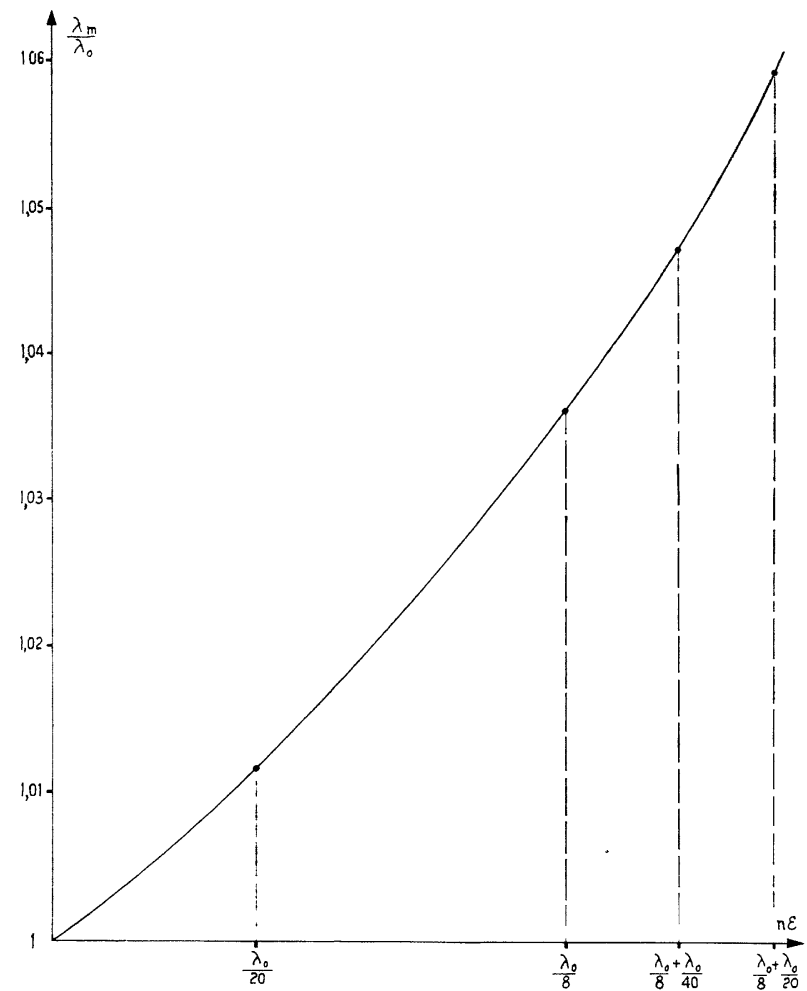

FIG. 3. - Longueur d'onde du maximum de $R$ en fonction de la surépaisseur optique $n \varepsilon$.

suffisante et l'on peut mesurer facilement un déplacement de $2 \AA$ du centre de la bande passante.

Dans ces conditions, la sensibilité du maximètre est largement suffisante pour mesurer avec précision des surépaisseurs de l'ordre de $\lambda_{0} / 200$. Le dépôt des surépaisseurs sur le revêtement réflecteur (recouvert d'une couche $\left.\lambda_{0} / 8\right)$ correspond à un cas très favorable pour la mesure d'épaisseurs de couches aussi minces. Le même contrôle effectué sur les premières couches du revêtement serait beaucoup plus difficile, la sensibilité étant alors beaucoup plus faible.
Pour corriger la planéité des lames, nous admettons maintenant que la correction se fera le plus facilement possible si l'on effectue cette correction sur la dernière couche du revêtement réflecteur déjà recouverte d'une couche uniforme $\lambda / 8$.

Le principe de correction proposé n'est pas à l'abri de toutes critiques. En effet, une surépaisseur du revêtement réflecteur entraîne une perte de pouvoir réflecteur visible sur la figure 2 , donc une perte de finesse. Mais cette perte de finesse reste petite par rapport à la différence entre des revêtements constitués de 5 et 7 couches, et de l'ordre de grandeur des différences que l'on obtient entre deux miroirs traités séparément. En outre, comme les déphasages à la surface dépendent de la longueur d'onde, la correction calculée pour $\lambda_{0}$ n'est valable que dans un domaine spectral limité (environ $400 \AA$ ), mais suffisante pour de nombreuses applications.

Conclusion. - Nous nous sommes essentiellement attachés à mettre au point un procédé de contrôle pour la préparation de couches d'épaisseurs optiques différentes de $\lambda / 4$ et en particulier de couches extrêmement minces. En utilisant un maximètre on dispose de deux méthodes. Elles permettent toutes deux de contrôler avec précision des couches de l'ordre de $\lambda / 200$; l'une, très simple et très générale, utilise un verre témoin, avec les inconvénients que cela comporte (faible fidélité); l'autre nécessite des calculs préalables difficiles mais permet le contrôle sur le verre lui-même; la seconde présente les qualités nécessaires pour envisager de l'appliquer à l'amélioration de planéité des lames d'interféromètre.

Manuscrit reçu le $1^{\text {er }}$ juillet 1966.

Nous remercions le Professeur R. Chabbal, directeur du Laboratoire Aimé-Gotton, avec qui nous avons eu de fructueuses discussions au cours de ce travail.

\section{BIBLIOGRAPHIE}

[1] Gracomo (P.), J. Physique Rad., 1958, 19, 307-311. Rev. Optique, 1956, 35, 317 et 442.

[2] Badoual, (R.), Thèse 1965, Caen. J. Physique, 1964, 25, 268. Applied Optics, 1966, 5, 63.

[3] KOPPELMANN (G.), VOSSKÜHLER (W.), Optik, 1965, 23, 181 .
[4] KoppelmanN (G.), KREBS (K.), Optik, 1961, 18, 349.

[5] Pelietier (E.), Chabbal (R.), Giacomo (P.), J. Physique, 1964, 25, 275.

[6] AbELìs (F.), Ann. Physique, 1948, 3, 504.

[7] PELLETIER (E.), Thèse 3 e Cycle C.N.R.S., Laboratoire A.-Cotton, Bellevue, France. 